\title{
BMJ open The trajectory to diagnosis with pulmonary arterial hypertension: a qualitative study
}

\author{
Ian Armstrong, ${ }^{1}$ Nikki Rochnia, ${ }^{2}$ Carl Harries, ${ }^{3}$ Sarah Bundock, ${ }^{4}$ Janelle Yorke ${ }^{2}$
}

To cite: Armstrong I, Rochnia N, Harries C, et al. The trajectory to diagnosis with pulmonary arterial hypertension: a qualitative study. BMJ Open 2012;2: e000806. doi:10.1136/ bmjopen-2011-000806

- Prepublication history for this paper is available online. To view these files please visit the journal online (http:// dx.doi.org/10.1136/ bmjopen-2011-000806).

Received 5 January 2012 Accepted 6 March 2012

This final article is available for use under the terms of the Creative Commons Attribution Non-Commercial 2.0 Licence; see http://bmjopen.bmj.com

\footnotetext{
${ }^{1}$ Department of Pulmonary Vascular Disease, Royal Hallamshire Hospital, Sheffield, UK

${ }^{2}$ School of Nursing, Midwifery \& Social Work, University of Salford, Manchester, UK ${ }^{3}$ Department of Pulmonary Vascular Disease, Royal Brompton and Harefield NHS Foundation Trust, London, UK

${ }^{4}$ HealthEd Group, Knutsford, Cheshire, UK
}

Correspondence to Dr Janelle Yorke; j.yorke@salford.ac.uk

\begin{abstract}
Objectives: To investigate the patient's experience of the trajectory to receiving a diagnosis of pulmonary arterial hypertension (PAH) and inform the provision of care for this patient group.

Design: Qualitative study using in-depth one-to-one interviews and pictorial representations. Data were analysed using thematic analysis.

Setting: Participants were interviewed in their own homes across England.

Participants: 30 patients with a diagnosis of pulmonary hypertension (18 participants were women, mean age 56 and range $26-80$ years and time since diagnosis ranged from a few months to more than 12 years) participated.
\end{abstract}

Results: All participants, regardless of the time since diagnosis, vividly described the process from manifestation of symptoms to receiving a confirmed diagnosis. The authors present data using three major themes: (i) making sense of symptoms, (ii) process of elimination and (iii) being diagnosed with PAH. Making sense of symptoms represented an early period of perseverance-people tried to carry-on as usual despite 'unexplained breathlessness'. As time progressed, this period was punctuated by critical events that triggered seeking medical advice. Once medical contact had been made, patients described a period of 'elimination' and convoluted contact with the medical profession. Dyspnoea misdiagnosis was a key factor that delayed the PAH diagnosis. Diagnosis disclosure by some medical professionals was also viewed as lacking empathy. More positive experiences were relayed when the medical team disclosing the diagnosis acknowledged previous limitations.

Conclusions: A lack of awareness of this illness from both the sufferer themselves and the medical profession emerged as a central theme and led to prolonged periods of being misdiagnosed. The application of a diagnostic pathway for unexplained dyspnoea that alerts practitioners to rare conditions could expedite the process of correct diagnosis.

\section{INTRODUCTION}

Pulmonary arterial hypertension (PAH) is a rare life-limiting disease that can progress rapidly. Living with a life-limiting illness can

\section{ARTICLE SUMMARY}

Research questions

- What is the patient's experience to receiving a diagnosis of pulmonary hypertension?

- What are the key patient and healthcare professional factors that prolong diagnosis?

\section{Key messages}

- There is a lack of awareness of this illness from both the sufferer themselves and the medical profession.

- A diagnostic pathway for unexplained dyspnoea may assist professionals to increase initial suspicion that less known conditions, such as $\mathrm{PAH}$, may be present.

- The pre-diagnostic journey can have long lasting effects on the individual and should form part of the patient's care pathway following referral to the specialist PAH centre.

Strengths and limitations of this study

- This is the first study to explore the PAH prediagnostic journey. Data were collected using indepth interviews incorporated with a novel approach of collecting pictorial representations. Given the role that medical professionals play in the patients' trajectory to diagnosis, their inclusion would be warranted in future studies.

present many challenges. Only two studies have previously investigated the experience of living with PAH from the patient's perspective; both reported the challenge of coping with uncertainty and living with invasive treatment regimens. ${ }^{1}{ }^{2}$ With the availability of effective therapy, early disease detection is an important strategic objective to improve patient outcomes. ${ }^{3} \mathrm{PAH}$ can be idiopathic, familial or associated with a number of conditions such as portal hypertension, connective tissue disease or exposure to drugs and toxins. ${ }^{4}$ This can make the process of diagnosing PAH challenging.

There is speculation that PAH may not be as rare as reported, but rather it is under diagnosed. ${ }^{5}$ Patients with $\mathrm{PAH}$ generally present with a spectrum of symptoms that 
may be attributed to general deconditioning or other cardiopulmonary conditions. Exertional dyspnoea is the most frequent presenting symptom, present in approximately $60 \%$ of patients and is eventually present in virtually all patients as the disease progresses. ${ }^{6}$ Fatigue and general exertion intolerance are also common complaints. Assessment of PAH is based on a logical sequence of determining whether there is a risk of PAH being present, whether PAH is likely to be present based on initial non-invasive evaluation and clarifying the underlying aetiology of PAH in an individual patient. ${ }^{7}$ Unfamiliarity with the condition by the general public and medical profession delays initial suspicion that PAH is likely to be present.

On average, the time period between the onset of the first symptoms to confirmed diagnosis of PAH ranges from 1 to 4 years. ${ }^{8}{ }^{9}$ Patient delay is most notably due to dyspnoea on exertion that is often attributed to deconditioning. ${ }^{8}$ Typically, patients are often seen by numerous doctors and receive incorrect diagnoses before a confirmed diagnosis of PAH is made. A survey conducted in the UK $(\mathrm{N}=488)$ showed that healthcare professional delay possesses a significant challenge: $44 \%$ of respondents were reviewed by four or more doctors before being correctly diagnosed and over a third experienced a delay of two or more years between seeing a doctor for the first time and being diagnosed. ${ }^{9}$

Reasons for patient and professional diagnostic delay in PAH are poorly understood. Anderson's model of Total Patient Delay ${ }^{10}$ provides detailed stages of a patient's pre-diagnostic pathway from awareness of a symptom to diagnosis and treatment. The model stipulates that people experience a series of stages, each governed by a conceptually distinct set of decisional and appraisal processes. The model has been applied to gain a greater understanding of delayed diagnosis of different types of cancer. ${ }^{11-13}$ In this study, we applied Anderson's model as a conceptual guide to understanding the trajectory to diagnosis with PAH. There are likely to be psychological consequences for patients and their families as a result of delayed diagnosis and access to appropriate services and support. The pre-diagnostic moments on the illness trajectory were important to people with cancer and influenced levels of satisfaction with subsequent care. ${ }^{14}$

This paper reports the data obtained from a programme of research exploring the experience of living with PAH. The focus of this report is the trajectory from symptoms first manifesting to receiving a confirmed diagnosis of PAH.

\section{METHODS}

Given the paucity of research and exploratory nature of the enquiry, a qualitative research approach was adopted. In-depth semistructured interviews were utilised to capitalise on the intricacies of the experiences of the participants. A semistructured interview style provides the flexibility to ensure that key topics are covered while enabling the interviewee to disclose information that may diverge from the interview guide. The aim of the interviews was to explore participants' experiences of living with this rare condition. Throughout the interviews, it became apparent that the pre-diagnostic phase represented a turbulent and poignant period for participants. This was also evident in the pictorial representations where participants focused largely on their experiences during the pre-diagnostic period. This was an unexpected finding. The decision was made to extract data that reflected the trajectory to receiving a confirmed PAH diagnosis and is presented in this paper.

\section{Participants}

Participants were recruited from the Pulmonary Hypertension Association UK database. The Pulmonary Hypertension Association is the only charity in the UK especially for people with pulmonary hypertension with approximately 3000 members. Members attending the 2009 PHA UK annual conference were informed about the project, and contact details were collected from those who expressed an interest in participating. We purposively sampled from this list to ensure maximal variation including both men and women with different ages, a range of aetiologies and time since diagnosis and different geographical areas in England. The study interviews were conducted in 2010 and took place in the patient's home. Thirty one-to-one in-depth interviews were conducted with patients with PAH. Details of the 30 patients are provided in table 1. Informed written consent was obtained from all participants at the beginning of the study and reviewed verbally at the time of interview. All participants provided informed written consent. Ethical approval was obtained from the Pulmonary Hypertension Association Medical Committee (01-2011) and the University of Salford Ethical Committee (NURA018).

\section{Data collection and analysis}

The in-depth interview guide was designed to cover the patient's experience of living with PAH (see box 1 for topic areas). Interviews were conducted by an experienced health researcher (SB) in participant's homes, and each lasted between 40 and $100 \mathrm{~min}$. At the beginning of the interview, participants were also asked to map put their 'individual journey' using a pictorial representation that included a straight line anchored with the words 'Before I had PAH' and 'Now'. This approach afforded participants with the opportunity to frame their own experiences. ${ }^{15} 16$ In this current study, the drawing technique was presented to participants as a timeline to assist participants to organise information and analyse the impact of previous experiences on current life. ${ }^{17}$ Interviews were audio-recorded and transcribed verbatim.

The method of thematic analysis described by Braun and Clarke ${ }^{18}$ was used to analyse the data collected. This method was employed to enable a flexible approach for 
Table 1 Characteristics of the 30 patients with pulmonary arterial hypertension interviewed for the study

\section{Number of patients $(\mathrm{N}=30)$}

\begin{tabular}{|c|c|}
\hline Sex (female:male) & $18: 12$ \\
\hline Age (mean years (SD, range)) & $56.3(38,2680)$ \\
\hline \multicolumn{2}{|l|}{ Working status } \\
\hline Work full time & 2 \\
\hline Work part time & 4 \\
\hline Retired & 11 \\
\hline Long-term sick & 9 \\
\hline Unemployed & 2 \\
\hline Education full time & 1 \\
\hline Education part time & 1 \\
\hline \multicolumn{2}{|l|}{$\mathrm{PAH}$ aetiology } \\
\hline Idiopathic & 11 \\
\hline Connective tissue disease & 7 \\
\hline Congenital heart disease & 5 \\
\hline Chronic thromboembolic & 2 \\
\hline Heritable & 1 \\
\hline Portal hypertension & 1 \\
\hline Lung disease & 1 \\
\hline Drugs/toxins & 1 \\
\hline $\begin{array}{l}\text { Duration of symptoms prior to } \\
\text { seeking medical advice (mean time) }\end{array}$ & 1 year, 9 months \\
\hline \multicolumn{2}{|l|}{ Time since $\mathrm{PAH}$ diagnosis } \\
\hline$<1$ year & 2 \\
\hline 1 to $<3$ years & 2 \\
\hline 3 to $<5$ years & 7 \\
\hline 5 to $<10$ years & 12 \\
\hline$>10$ years & 7 \\
\hline \multicolumn{2}{|l|}{ NYHA classification } \\
\hline I & 0 \\
\hline II & 9 \\
\hline III & 19 \\
\hline IV & 2 \\
\hline
\end{tabular}

analysis of the interview transcripts and the pictorial data. In addition, participants tended to tell their stories chronologically and thematic analysis enabled themes that represented key milestones in the trajectory from

\section{Box 1 Interview guide pertinent to this paper}

- Participants were asked to map out their journey showing all the highs and lows, and the key events along the way, via pictorial representations. A range of stimulus materials were provided (figures 1 and 2).

- Participants were encouraged to discuss their journey while completing their picture.

- The following questions relevant to this paper were asked:

- When you were first diagnosed what were your thoughts and feelings?

- How do you feel about pulmonary hypertension now?

- In what ways, if any, have your perceptions regarding pulmonary hypertension changed since you were first diagnosed?
pre-PAH to post-PAH diagnosis to form the basis of the data reported in this paper. Data analysis was conducted by NR and SB and JY conducted a review of the audit trail to confirm validity of the themes extracted.

\section{RESULTS}

The characteristics of the 30 patients recruited to the study are detailed in table 1 . The coding used to represent participants is as follows: $\mathrm{P}=$ participant; $\mathrm{F}=$ female, $\mathrm{M}=$ male; individual study code; participant's age (eg, PF2:56 is a female patient, study code 2 and 56 years of age).

All participants, regardless of the time since diagnosis, vividly described the process from initially experiencing subtle symptoms to receiving a confirmed diagnosis of $\mathrm{PAH}$, both verbally and via a pictorial representation. Participants seemed to have a 'need' to recount their pre-diagnostic journey, and it was from this that the data set of the patient's pre-diagnosis journey was extracted. Due to the length of time it took for an eventual diagnosis, the plethora of medical consultations and tests and consequent emotional roller coaster, the road to diagnosis was strewn with feelings of 'frustration', 'anger' and 'uncertainty' for both the patient and their family. This is illustrated in the pictorial representations-these tended to focus mostly on the pre-diagnostic period as opposed to post-diagnosis (figures 1 and 2). We present data using three major themes that represent the chronological stories relayed by participants: (i) making sense of the symptoms, (ii) the process of elimination and (iii) being diagnosed with PAH: 'It's worse than cancer; we can't treat it'.

Research participants were requested to use the paper and drawing utensils to map out their journey on the timeline from 'Before I had PH' to 'Now'. The participants were provided with a range of resources including stickers that represented a range of facial expressions and emotions and different clinical tests. Information placed above the timeline was described as representing 'good' experiences and information placed below as representing 'bad' experiences.

\section{Symptom appraisal}

\section{Explanations considered accounting for the symptoms} experienced

Many participants did not visit a doctor until they had symptoms for over a year. Participants were able to provide detailed descriptions of symptoms that were experienced for extended periods of time including breathlessness, palpitations, chest pain, haemoptysis and oedema and associated weight gain, all known to be associated with $\mathrm{PAH}^{4}{ }^{5} \mathrm{~A}$ process of appraising the presence and cause of symptoms was apparent (Anderson's stage 1, see figure 3). Although generally acknowledged as 'gradually creeping on', breathlessness was salient though meant many different things: 'just getting unfit' [PF2:56; PF26:52], 'getting old' [PM08:70] and 'I've always had ... things like flu' [PF19:34]. Participants who had recently given up smoking around 


\section{pha G}

\section{My PH journey}

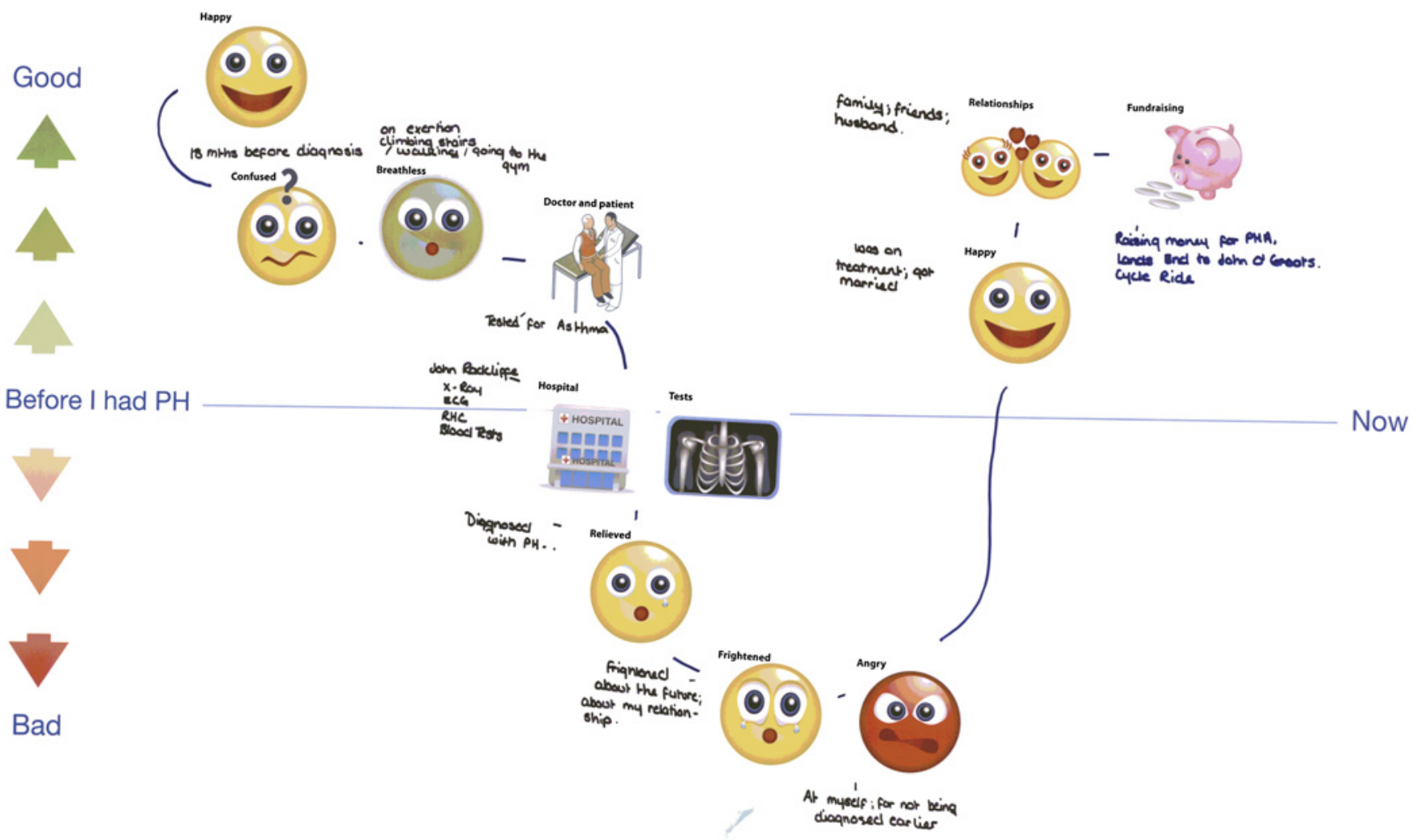

Figure 1 Research participants were requested to use the paper and drawing utensils to map out their journey on the timeline from "Before I had PH" to "Now". The participants were provided with a range of resources including stickers that represented a range of facial expressions and emotions, and different clinical tests. Information placed above the timeline was described as representing "good" experiences and information placed below as representing "bad" experiences.

the same time as experiencing symptoms 'just assumed that everything was down to smoking' [PF26:52] and that 'people get short of breath once they give up smoking' [PF19:34].

A period of perseverance was described by participants as they attempted to rectify their perceived lack of fitness. Younger participants in particular told stories of taking unknown risks and 'just carried on regardless' [PF14:35], leading to a delayed process of symptom interpretation. 'I was getting really out of breath ... on the step machine or running machine ... very quickly, but couldn't understand why. I was going down there two or three times a week ... I'm thinking ... I should be getting fitter' [PF7:35].

Alternatively, some participants used avoidance strategies and adapted activities according to the severity of the breathlessness they experienced: 'I was late for the tram ... I decided to run for it. I was astonished to find that as I got onto it, I felt really out of breath and really ill ... it was great shock ... I decided; perhaps I won't run for the tram in future. At that point I did nothing about it' [PM11:63]. The decision to seek medical opinion was delayed as participants 'knew something was wrong ... but ... didn't want to' [PF19:34]. Covering-up of symptoms became the norm in an attempt to protect both oneself and family members: 'I remember thinking - I'm going to have to sort of stop and pretend I'm looking at traffic' [PF5:63] and 'I started to think, this is really bad ... I hid it all the time.' [PF14:35]. This frequently led to a delay in time from the person concluding that he or she had a serious health problem to deciding to seek professional help (Anderson's stage 2).

\section{Trigger for seeking medical advice}

Perceived seriousness of the physiological condition by either the patient or a family member was the catalyst required for medical contact to be made (Anderson's stages 3 and 4). All participants recalled the time when they first decided to seek medical advice, and this often involved a dramatic description where either the patient themselves or a family member or friend initiated seeking medical advice (box 2).

\section{Process of elimination}

'They can only eliminate everything else and then it's got to be pulmonary hypertension' [PM25:54]. 


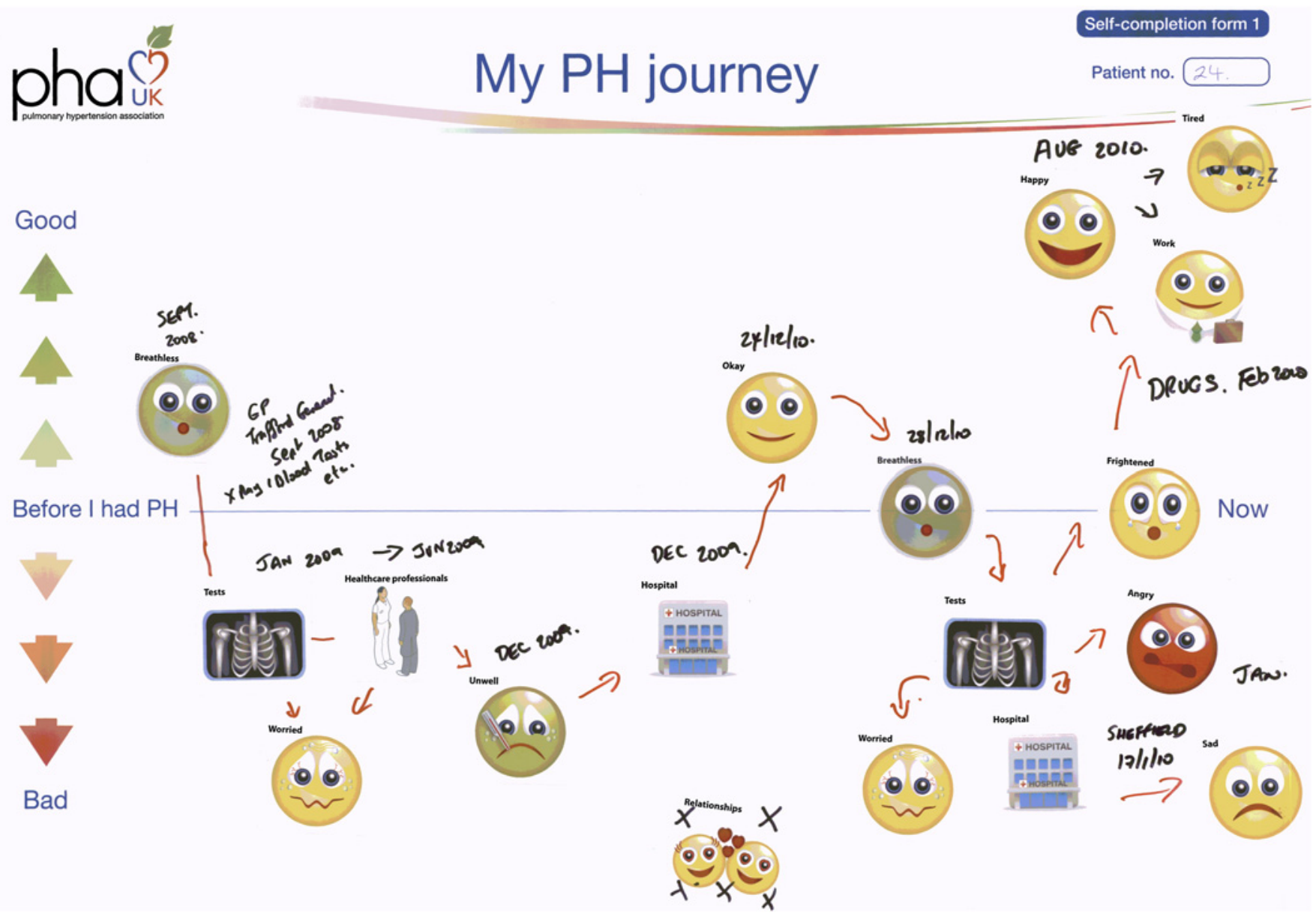

Figure 2 Research participants were requested to use the paper and drawing utensils to map out their journey on the timeline from "Before I had PH" to "Now". The participants were provided with a range of resources including stickers that represented a range of facial expressions and emotions, and different clinical tests. Information placed above the timeline was described as representing "good" experiences and information placed below as representing "bad" experiences.

\section{Convoluted contact with medical professionals}

The first visit to the doctor by no means guaranteed a diagnosis; most participants had to see four or more

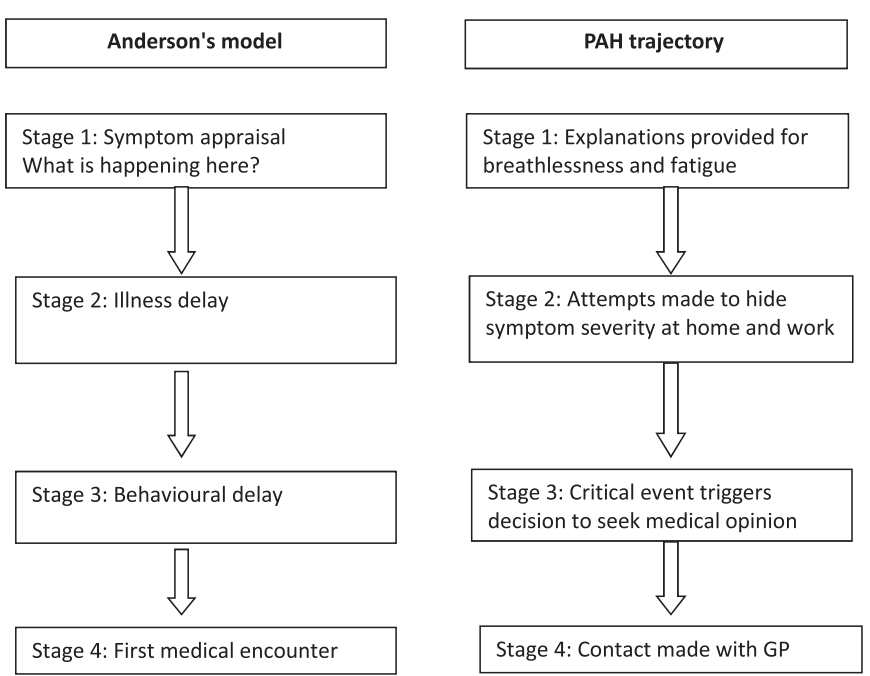

Figure 3 Anderson's model of 'total patient delay' and its relationship to pulmonary arterial hypertension. doctors before they were correctly diagnosed with PAH. Any particular symptom could have been due to the subsequent diagnosis or to some other condition, especially if months or years had passed between symptom presentation and diagnosis. All participants went through a phase of visiting their GP but to no avail: 'he [GP] didn't know what I was talking about. He couldn't find a thing and I was just getting worse' [PF20:75]. One young participant described her "lowest point-everything had gone wrong ... they [GP'] couldn't explain the swelling, my heart rate was incredibly high, I couldn't breathe, had low blood pressure." Misdiagnosis by the GP and numerous specialists that patients were referred to was common (box 3).

Throughout the telling of their histories, participants spoke of how they felt that clinicians 'didn't recognise pulmonary hypertension' [PM8:80] and had little understanding of the diagnostic indicators. All participants described undergoing a battery of tests either requested by the GP or by one of the numerous specialists that they were referred to, with many of the tests being repeated on multiple occasions: 'He'd just no idea what it was so he just kept putting me on 


\section{Box 2 Examples of triggers for seeking medical advice}

Patient's self-recognition of trigger

- 'I started blacking out. That's when I went to see the doctor. I thought maybe I was diabetic' [PF14:35].

- 'I walked up a short ladder and suddenly felt breathlessness. I thought ... what's going on now' [PM25:54].

Family/friend recognition of trigger

- 'He [partner] thought I was being attacked [while talking on the phone] ... I was so out of breath' [PF19:34].

- 'I met a girl that used to be a nurse [in the street] ... I stopped, I could not even speak to her ... she says to me what's wrong with you you're not well ... I says I haven't a breath ... she says ... you shouldn't be like that [PF20:75]

n 'I came home for the summer. My mum's a nurse, so it was when she started noticing that I couldn't walk, never mind breathe ... so she sent me to the doctors' [PF29:26].

- 'Everyone thought I was pregnant because I was putting on lots of weight ... my mum picked me up after my shift, and I told her that my feet had swollen up, so she said, 'Well see if they go down, if they haven't l'll take you to the hospital later.' Everything below my knees was huge, they'd gone like tree trunks. So she took me straight to the hospital' [PF19:34].

running machines ... coloured stuff in my veins. In the end, he said I don't think we're getting anywhere' [PF14:35].

Participants were subsequently referred from one specialist to another: 'it was the cardiac specialist, respiratory specialist, Warfarin specialist, radiographer, a case conference' [PF29:26]. The lack of explanation or diagnosis led to feelings of anger and frustration: "when I was sent home I was fuming ... when they're telling you that there's nowt [nothing] wrong with you and you know there is' [PM3:47]. Participants became 'disillusioned ... [I was thinking] there must be something wrong and somebody isn't recognising what it is' [PM8;80]. Delays or concerns that their symptoms were not being taken seriously sometimes also prompted participants to use private healthcare services.

\section{Life interrupted}

Given the lack of a medical diagnosis, participants tried to carry on with family and work life as normal, despite worsening symptoms. One participant explained how she would weed her garden lying on the ground because 'I couldn't breathe if I bent down' [PF13:65].

Having no apparent medical reason for being so ill made working difficult too: 'work didn't like it - 'nobody gets a cold that often'-I was being bullied' [PF26:56] and 'sick notes, I actually had a reputation for being ... a bit ... illy [sick] type person. I hated it' [PF19:34]. Misconceptions from work colleagues who thought 'well, you should be better by now' [PF29:26] also led to participants trying to hide their symptoms at work.

\section{Box 3 Examples of misdiagnosis}

- 'I was given an inhaler. It made no difference whatsoever and after a few months he [GP] thought it might be stress, panic attacks ... so prescribed [anxiolytic] ... but it didn't do anything for my breathlessness' [PM11:63].

- 'They [GP] thought it was antiphospholipid syndrome for quite a while and lupus, but none of the tests came back positive' [PM25:54].

- 'All of this time I was getting more and more asthma medication' [PF29:26].

- 'They [patient's history presented at a case-conference] thought it might be the pill' [PF29:26].

n 'He [respiratory specialist] twigged straight away that can't just have asthma. So this was going on for six years' [PM12:31].

n 'I just wanted answers-it was getting ridiculous' [PM08:70].

\section{Being diagnosed with pulmonary hypertension}

'It's worse than cancer, we can't treat it' [PF14:35].

\section{Diagnosis disclosure}

Participant's recounts of the time when they were told that they had PAH described a mix of emotions: 'a whirl; good and bad' [PF11:20]. Some patients described inappropriate disclosure from non-medical staff: 'I knew something was wrong because she was shouting for the cardiologist to come and look at the echo. I was lying there thinking; something's not right here and that is when the panic set in' [PF19:34]. Another was told by a social worker that she 'had an inoperable condition and that I now needed to consider myself as disabled. I just cried. I was distraught' [PF2:56].

Disclosure by some medical professionals was also viewed as lacking empathy. One explained that she was told 'you've got this, it's worse than cancer, we can't treat it, can't have a heart-lung transplant, we need to cut half your heart and we're going to do that on Monday ... that's when I nearly died' [PF14:35]. More positive experiences were relayed when the team disclosing the diagnosis acknowledged previous limitations: 'they [medical team] were terribly apologetic and said, we never dreamt it was this and we are so sorry that it's taken us so long to get this far', although gaps remained 'they didn't know why I had it ... what had caused it at the time' [PF2:56].

\section{Making sense of the diagnosis}

The diagnosis of PAH evoked a range of emotions, and for most participants, it was the first time they had heard of the condition. There was a mix of 'feeling relief but scared' [PM3:47]. For some, there was relief that the diagnosis was not psychosomatic: 'before, everybody thought it was because of the depression' [PM10:61].

Predominately, participants felt ill informed about their condition and many turned to the internet as a source of information gathering, often with devastating 
Box 4 Examples of making sense of the diagnosisspecialist pulmonary arterial hypertension contact

- '... people were doing things, explained what different tests actually meant ... drugs explained, right at the start in the hospital' [PF13:65].

- 'We were told there were lots of medications these days that could help and people were doing quite well on them. It wasn't as devastatingly unhopeful as first appeared on the internet' [PF2:56].

- 'I felt better because they told me what was up' [P3M:47].

- 'They understood ... it was like, finally you get what I'm going through ... could explain all the hard questions-'It's not a death sentence, there's numbers of reasons why you could have it ... stay here and we'll find out why and we'll put you on the best treatment' [PF29:26].

consequences: 'every testimonial we read, the person had died from it' [PF29:26] and 'you're only going to live 2 years' [PF2:56; PF6:69; PF29:26]. However, reassurance was provided during the initial visits at $\mathrm{PAH}$ specialist centres where most felt that they were informed of what PAH is and what treatment options are available (see box 4 for examples).

Resentment and anger became evident when participants reflected on the extended time that it took for a definitive diagnosis (figures 1 and 2) and whether 'if I'd been diagnosed earlier perhaps I wouldn't have been so bad' [PF21:65]. It was suggested that 'that was the whole problem, it had gone on so long' [PF23:72]. Although it was acknowledged that PAH cannot be tested for per se- they can only eliminate everything else and then it's got to be PAH' [PM25:54].

\section{DISCUSSION}

\section{Summary of main findings}

To our knowledge, this is the first report of the patient's trajectory to receiving a confirmed diagnosis of PAH. In this study, a lack of awareness of this illness from both the sufferer themselves and the medical profession emerged as a central theme of the trajectory to a confirmed diagnosis. People appeared to attempt to make sense of unexplained breathlessness through rationalising of potential reasons until a significant event triggered consultation with a medical professional. Participants recounted a serious decline in their health followed by a dramatic event such as collapsing in the street or family members pleading with them to seek medical advice. What followed was an extended period of contact with few answers, while the condition continued to deteriorate. This made family and work life difficult, and conflict was often described as having taken place in the workplace as a result. Asthma was a common misdiagnosis, and participants underwent several tests at their local hospital but PAH was not suspected at the time. Diagnosis disclosure was mostly viewed by participants as lacking in sensitivity. The protracted journey left some with a sense of anger and wondering if an expedited diagnosis may have improved their response to treatment and prognosis. Internet searching for information related to $\mathrm{PAH}$ increased anxiety and uncertainty which was only eased when under the care of a specialist centre.

\section{Meaning and implications}

The data presented here elucidate the reasons why some people with $\mathrm{PAH}$ experience delays in obtaining a diagnosis of PAH. The pre-diagnostic phase is punctuation by transitional stages that are reflected in Anderson's model of total patient delay. ${ }^{10}$ Participants described a period of time where symptoms were apparent, in particular breathlessness, but a range of self-explanations were suggested such as increasing age and poor level of fitness. These patient-attributed delays are similar to those found with the insidious onset of other long-term conditions, notably chronic obstructive pulmonary disease. $^{19}{ }^{20}$ However, unlike chronic obstructive pulmonary disease, which has been described as 'a story with no beginning ${ }^{19}$ PAH patients could vividly recall the onset of symptoms. It is difficult to make direct comparisons with other conditions that present with salient symptoms and misdiagnosis because PAH affects a wide range of ages. In our study, the age of participants ranged from 26 to 80 years. We opted to include such a wide age group due to the dearth of research relating to the experiences of people living with this rare disease. We found that the pre-diagnostic experience of patients, including symptom interpretation and responses from healthcare professionals, differed according to age.

Participants in this study were found to have experienced significant illness and behavioural delay (stages 2 and 3 of Anderson's model). The delay was apparent regardless as to the age of the individual. Younger and older participants described knowing that something was wrong but 'illness delay' as a result of their own perceptions continued until experiencing a critical event or making a medical appointment at the insistence of a family member or friend. It is likely that participants in this study did not perceive the symptoms of PAH as serious and were not aware of the potential consequences of delaying medical opinion.

Overreliance on the internet may have prolonged 'total patient delay'. In an attempt to cope with uncertainty, participants in our study were inclined to search the internet for answers including self-diagnosis and trying to understand the diagnosis of PAH. Information seeking by people diagnosed with $\mathrm{PAH}$ is related to uncertainty about prognosis and limited knowledge of the condition. ${ }^{2}$ With diffusion and popularity of the internet in recent years, it is inevitable that many people will use this mode of communication as a source of health-related information gathering. ${ }^{21}{ }^{22}$ In our study, people were often misinformed because there was overreliance on personal accounts of patients or family members living with $\mathrm{PAH}$-where accounts included 
a poor portrayal of life expectancy and negative narratives-rather than accessing more reputable sites (eg, the Pulmonary Hypertension Association UK and British Lung Foundation).

Anderson's model has been previously expanded to include healthcare professional delay in relation to obtaining a diagnosis of ovarian cancer ${ }^{11}$ and, as illustrated in this study, is also relevant to PAH. In the context of conditions such as ovarian cancer, delay by patients accounts for most of the delay experienced. ${ }^{11}$ However, our study suggests that professional delay-misdiagnosis, a battery of tests and referrals to different specialists-is significant and requires further investigation and attention. $\mathrm{PAH}$ is a diagnosis of exclusion ${ }^{7}$; consequently, delays attributed to the patient are often compounded by medical professional's delays because PAH is so difficult to diagnose. The vagueness and non-specificity of PAH symptoms challenge GPs, who may see only one PAH patient in their entire career. Patients present with a variety of symptoms, all of which could be caused by common pulmonary conditions. Since the symptoms are non-specific, the initial evaluation of patients with these symptoms is often appropriately directed at diagnosing or excluding more common conditions. In the absence of an identifiable explanation, however, pulmonary vascular disease should be considered as a possible cause for these symptoms, particularly unexplained dyspnoea.

In the UK, people who are suspected of having cancer, including cancer of the lung, can expect to see a specialist within 2 weeks. ${ }^{23}$ No national guidelines exist for unexplained dyspnoea where cancer is not suspected. Guidelines and consensus statements exist for the assessment and management of dyspnoea associated with diagnosed respiratory or cardiac conditions but these do not address the assessment of dyspnoea of unknown cause. ${ }^{24} 25$ A diagnostic pathway for unexplained dyspnoea may assist GPs and other healthcare professionals to increase initial suspicion that less known conditions, such as $\mathrm{PAH}$, may be present and require specific investigative testing. Active follow-up by GPs of all patients with uncertain diagnoses is unrealistic when NHS resources are stretched, but individuals should be encouraged to return to their GP if their symptoms persist ${ }^{11}$ and not made to feel like they are 'bothering' the doctor. Specifically, setting a time limit for the followup of people should leave no doubt about how long dyspnoea should go unchecked.

Most participants described encounters with health professionals that were perceived as lacking in empathy. However, breaking bad news is also a complex communication task. Bad news often must be delivered in settings that are not conducive to such intimate conversations. The hectic pace of clinical practice may result in a health professional delivering bad news with little forewarning or when other responsibilities are competing for attention. The complexity of the interaction can sometimes create serious miscommunications such as patient misunderstanding about the prognosis of the illness or purpose of care. ${ }^{26}$ Poor communication is associated with reduced patient satisfaction. ${ }^{27}{ }^{28}$ In the particular context of diagnostic and prognostic information, the crucial role of communication in 'breaking bad news' can have a significant negative influence on the patient's psychosocial experience, symptom management, treatment decisions and quality of life. ${ }^{14} 28-30$

Regardless as to the amount of time that had past, participants' pictorial representations of their PAH journey were largely focused on the pre-diagnostic period which included the use of stimulus materials that consisted of negative emotional connotations (eg, anger and frustration). One participant who expressed a positive experience both pre-diagnosis and post-diagnosis described how the medical team offered an apology for not diagnosing PAH early. These results suggest that the pre-diagnostic journey can have long-lasting effects on the individual with $\mathrm{PAH}$ and should form part of the patient's care pathway following referral to the specialist $\mathrm{PAH}$ centre. Referral to the specialist centre eased patients' uncertainty as questions were answered, and for most participants, there was a sense of relief that, despite initial perceptions, viable treatments for PAH were available. There are nine specialist pulmonary hypertension centres in the UK and Ireland which look after people with PAH. Specialists' centres adhere to international guidelines for the diagnosis and management of PAH. ${ }^{731}$ Participants in our study expressed concern about the length of time that it took to be referred to a specialists' centre; many believed that if they had been referred earlier, they would not have become so sick and would have a better response to treatment.

\section{STRENGTHS AND LIMITATIONS OF THIS STUDY}

This qualitative study aimed for a maximum variation sample and 'in-depth' description of diagnosis delay. As such, we included patients with a wide range of backgrounds and experiences and variation in length of time since being diagnosed with $\mathrm{PAH}$ and not just those who had particular concerns about diagnostic delay. We believe we have identified a wider range of diagnostic delays attributable to both the patient and healthcare professionals. Given the role that the medical profession play in the patients' trajectory to PAH diagnosis, the exclusion of their views represents a limitation to the current study. Living with a life-limiting condition can present many challenges and life stressors; therefore, caution is required in interpreting retrospective narrative interviews of an individual's illness trajectory. The data rely on patient's recall of events and their accounts of the pre-diagnostic phase involved. These accounts may benefit from hindsight and may or may not accurately reflect what really happened. Participants in our study ranged from $<1$ year to more than 10 years since diagnosis confirmation. It could be argued that the health service had changed during this time; however, we found that participants' reflections were 
similar and told in a vivid way despite the amount of time that had lapsed. Nonetheless, the stories told in this study reflect patients' current views and therefore provides a unique insight into people's perceptions and understandings of their experience and the meanings they attach to it.

\section{CONCLUSIONS}

Limited awareness of PAH outside specialist centres leads to misdiagnosis, delays in treatment and an increasing sense of anger, frustration and confusion for many patients. Repeated tests and visits to numerous medical professionals, including GPs and cardiac or respiratory consultants over a number of years, represents a significant use of resources and consequent cost to the NHS. National guidelines for the assessment and diagnostic pathway of unexplained dyspnoea are recommended.

Our results emphasise the importance of increasing awareness of this disease in order to expedite the process of correct diagnosis and the referral of patients with $\mathrm{PAH}$ to specialised centres. Voluntary organisations may wish to promote awareness of PAH and populate stories that represent the wide range of people that it can affect to aid both public and healthcare professional understanding of this life-limiting serious disease.

Acknowledgements The authors are grateful to all patients who took time to take part in this study.

Contributors Study conceptualisation and design: IA, CH, SB and JY; data analyses: NR, SB and JY and manuscript preparation: IA, NR, CH, CB and JY.

Funding Funded by Pulmonary Hypertension Association UK, grant number 2010-2011.

Competing interests JY received funding from Pulmonary Hypertension Association (UK) who funded this study, for workshops and attendance at medical conferences.

Ethics approval University of Salford and PHA UK Medical Board.

Provenance and peer review Not commissioned; externally peer reviewed.

Data sharing statement Unpublished data are available to Dr JY and PHA UK board of Trustees.

\section{REFERENCES}

1. Peloquin J, Robichaud-Ekstran S, Pepin J. Perception of quality of life by women with stage III or IV primary pulmonary hypertension and receiving treatment with prostacyclin. Can J Nurs Res 1998;8:113-36.

2. Flattery MP, Pinson JM, Savage L, et al. Living with pulmonary arterial hypertension: patient's experiences. Heart Lung 2005;34:99-107.

3. Lau EM, Manes A, Celermajer DS, et al. Early detection of pulmonary vascular disease in pulmonary arterial hypertension: time to move forward. Eur Heart J 2011;32:2489-98.

4. Simonneau G, Robbins IM, Beghetti M, et al. Updated clinical classification of pulmonary hypertension. J Am Coll Cardiol 2009;54 (1 Suppl):S43-54.

5. Matura LA, Carroll DL. Human responses to pulmonary arterial hypertension: review of the literature. J Cardiovasc Nurs 2010;25:420-7.

6. Humbert $\mathrm{M}$, Sitbon $\mathrm{O}$, Chaouat $\mathrm{A}$, et al. Pulmonary arterial hypertension in France: results from a National Registry. Am J Respir Crit Care Med 2006;173:1023-30.

7. Galie N, Hoeper MM, Humbert M, et al; ESC Committee for Practice Guidelines (CPG). Guidelines for the diagnosis and treatment of pulmonary hypertension: the task force for the diagnosis and treatment of pulmonary hypertension of the European Society of Cardiology and the European Respiratory Society, endorsed by the International Society of Heart and Lung Transplantation. Eur Heart $J$ 2009;30:2493-537.

8. Wilkens $\mathrm{H}$, Grimminger $\mathrm{F}$, Hoeper $\mathrm{M}$, et al. Burden of pulmonary arterial hypertension in Germany. Respir Med 2010;104:902-10.

9. Armstrong I, Harries C, Yorke J. The imPAHct survey: living with pulmonary arterial hypertension. Am J Respir Crit Care Med 2011: A6130.

10. Anderson BL, Cacioppo JT, Roberts DC. Delay in seeking a cancer diagnosis: delay stages and psychophysiological comparison processes. Br J Soc Psychol 1995;34:33-52.

11. Evans J, Ziebland S, McPherson A. Minimising delays in ovarian cancer diagnosis: an expansion of Anderson's model of 'total patient delay'. Fam Pract 2007;24:48-55.

12. Scott SE, Grunfeld EA, Main J, et al. Patient delay in oral cancer: a qualitative study of patients' experiences. Psychooncology 2006;15:474-85.

13. Walter FM, Humphrys E, Tso S, et al. Patient understanding of moles and skin cancer. BMC Fam Pract 2010;11:62.

14. Thorne SE, Bultz BD, Baile WF; SCRN Communication Team. Is there a cost to poor communication in cancer care: a critical review of the literature. Psychooncology 2005;14:875-84; discussion $885-6$.

15. Harrison B. Seeing health and illness worlds-using visual methodologies in a sociology of health and illness: a methodological review. Sociol Health IIIness 2002;24:856-72.

16. Kearney KS, Hyle AE. Drawing out emotions: the use of participantproduced drawings in qualitative inquiry. Qual Res 2004;4: 361-82.

17. Deacon SA. Creativity within qualitative research on families: new ideas for old methods. [35 paragraphs]. The Qualitative Report, Volume 4, Numbers 3 \& 4, March, 2000 (http://www.nova.edu/ssss/ QR/QR4-3/deacon.html)

18. Braun V, Clarke V. Using thematic analysis in psychology. Qual Res Psychol 2006;3:77-101.

19. Pinnock $\mathrm{H}$, Kendall M, Murray SA, et al. Living and dying with severe chronic obstructive pulmonary disease: multi-perspective longitudinal qualitative study. BMJ 2010;342:d142.

20. Price D, Freeman D, Cleland J, et al. Earlier diagnosis and earlier treatment of chronic obstructive pulmonary disease in primary care. Prim Care Respir J 2011;20:15-22.

21. Nguyen $\mathrm{HQ}$, Carrieri-Kohlman V, Rankin SH, et al. Internet-based patient education and support interventions: a review of evaluation studies and directions for future research. Comput Biol Med 2004;34:95-112.

22. Boyd K, Worth A, Kendall M, et al. Making sure services deliver for people with advanced heart failure: a longitudinal qualitative study of patients, family carers, and health professionals. Palliat Med 2009;23:767-76.

23. National Institute for Health and Clinical Excellence. Referral Guidelines for Suspected Cancer. Clinical Guideline 27. London: National Institute for Health and Clinical Excellence, 2005.

24. Mahler DA, Selecky PA, Harrod CG, et al. American College of Chest Physicians consensus statement on the management of dyspnea in patients with advanced lung of heart disease. Chest 2010;137:674-91.

25. American Thoracic Society. Dyspnea mechanisms, assessment, and management: a consensus statement. Am J Respir Crit Care Med 1999;159:321-40.

26. Quirt CF, McKillop WJ, Ginsberg AD, et al. Do doctors know when their patients don't? A survey of doctor-patient communication in lung cancer. Lung Cancer 1997;18:1-20.

27. Ong LM, Visser MR, Lammes FB, et al. Doctor-patient communication and cancer patients' quality of life and satisfaction. Patient Educ Couns 2000;41:145-56.

28. Zachariae R, Pedersen CG, Jensen AB, et al. Association of perceived physician communication style with patient satisfaction, distress, cancer-related self-efficacy, and perceived control over the disease. Br J Cancer 2003;88:658-65.

29. Lobb EA, Butow PN, Kenny DT, et al. Communicating prognosis in early breast cancer: do women understand the language used? Med J Aust 1999;171:290-4.

30. Parker PA, Baile WF, de Moor C, et al. Breaking bad news about cancer: patients' preferences for communication. J Clin Oncol 2001;19:2049-56

31. National Pulmonary Hypertension Centres of the UK and Ireland. Consensus statement on the management of pulmonary hypertension in clinical practice. Heart 2008;94(Suppl 1):i1-41. 